\title{
Terminal-Specific Interaction between Double-Stranded DNA Layers: Colloidal Dispersion Behavior and Surface Force
}

Naoki Kanayama, ${ }^{*},+\neq \odot$ Taito Sekine, ${ }^{\S}$ Kazunari Ozasa, ${ }^{*}$ Satomi Kishi, ${ }^{\ddagger}$ Takashi Nyu, ${ }^{\S}$

Tomohiro Hayashi, ${ }^{*}$, and Mizuo Maeda

${ }^{\dagger}$ Interdisciplinary Graduate School of Science and Technology, Shinshu University, 4-7-1 Wakasato, Nagano, Nagano 380-8553, Japan

${ }^{\ddagger}$ Bioengineering Laboratory, RIKEN, 2-1 Hirosawa, Wako-shi, Saitama 351-0198, Japan

${ }^{\S}$ Department of Materials Science and Engineering, School of Materials and Chemical Technology, Tokyo Institute of Technology, 4259 Nagatsuta-cho, Midori-ku, Yokohama, Kanagawa 226-8502, Japan

Supporting Information

ABSTRACT: Double-stranded DNA-grafted nanoparticles (dsDNA-NPs) exhibit a unique dispersion behavior under high-salt conditions depending on the pairing status of their outermost base pairs (pairing or unpairing). The dsDNA-NPs having complementary (i.e., pairing) outermost base pairs spontaneously aggregate under high-salt conditions, but not when their outermost base pairs are mismatched (unpairing). In this study, we used colloidal probe atomic force microscopy to examine how the outermost base pairs affect the interaction between the dsDNA-grafted layers (dsDNA layers). To precisely assess the subtle structural differences in the dsDNA layers, we developed a method for the formation of a homogenous dsDNA layer on gold surfaces using hairpin-

Terminal-Specific Interaction under High-Salt Conditions

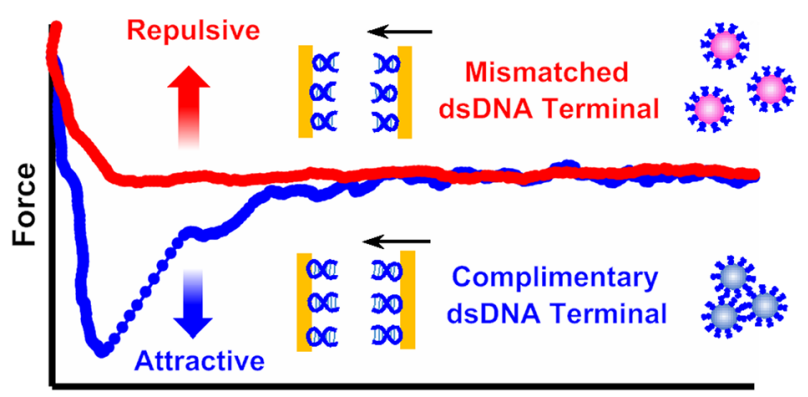

Distance shaped DNAs. Homogenous dsDNA layers having complementary $(\mathrm{G}-\mathrm{C})$ or mismatched $(\mathrm{C}-\mathrm{C})$ outermost base pairs were grafted onto the surfaces of colloidal probes and gold substrates. Force-distance curves measured in an aqueous medium under high-salt conditions revealed that the surface forces between the dsDNA layers were bilateral in nature and were governed by the outermost base pairs. Between complementary outermost dsDNA layers, the surface force changed from repulsive to attractive with an increase in the $\mathrm{NaCl}$ concentration $(10-1000 \mathrm{mM})$. The attraction observed under high-salt conditions was attributed to the site-specific interaction proceeded only between complementary dsDNA terminals, the so-called blunt-end stacking. In fact, between mismatched outermost dsDNA layers, the repulsive force was mostly dominant within the same $\mathrm{NaCl}$ concentration range. Our results clearly revealed that the pairing status of the outermost base pairs has significant implications for the surface forces between dsDNA layers, leading to the unique dispersion behavior of dsDNA-NPs.

\section{INTRODUCTION}

The properties of DNA strands immobilized at a solid-liquid interface have received considerable attention in fundamental and applied research because they play key roles in various DNA-related functional devices, including biosensors. ${ }^{1-5}$ The formation of DNA layers on solid surfaces often results in restricted flexibility of the conformation of DNA strands because of crowding molecular packing (steric hindrance) and an aligned configuration of DNA on the surface. In addition, the local ionic environment (ionic strength and composition) surrounding the DNA layer affects the physicochemical characteristics of the surface-immobilized DNA strands, yielding characteristics very different from those of free DNA in solution. ${ }^{6-9}$ For example, it has been reported that singlestranded DNA (ssDNA) that is densely immobilized on gold nanoparticles (GNPs) [spherical nucleic acids (SNAs) ${ }^{10}$ ] shows a higher affinity for complementary DNA than the same ssDNA that is free in solution. ${ }^{11,12}$ Moreover, the melting transition of DNA duplexes formed on the SNA surface or cross-linked between the SNAs occurs at a higher and narrower temperature range compared with that of free DNA duplexes of the same sequence. ${ }^{13,14}$

More than 10 years ago, we identified a unique dispersion behavior of DNA-immobilized NPs, that is, the dispersion state of double-stranded DNA-grafted nanoparticles (dsDNA-NPs) under high-salt conditions is governed exclusively by the pairing status of the outermost base pairs (base pairing or unpairing). ${ }^{15}$ The dispersion of dsDNA-NPs having complementary (i.e., pairing) outermost base pairs shows a non-cross-linking aggregation response under high-salt conditions, but the dispersion of dsDNA-NPs having mismatched (i.e., unpairing) outermost base pairs does not. Interestingly, this behavior is

Received: September 21, 2016

Revised: November 3, 2016

Published: November 7, 2016 
generally observed irrespective of the material (gold and polymers), ${ }^{15-17}$ size, ${ }^{17-19}$ and shape (sphere, rod, triangle) $)^{20}$ of the particle core, and it is also independent of the base sequence of the inner region of dsDNA-grafted layers (dsDNA layers). ${ }^{15}$ These observations reveal that a single-base pairing or unpairing at the outermost surface of the dsDNA layer on NPs dominates the aggregation or dispersion of dsDNA-NPs, although the underlying mechanism of this phenomenon is still unclear. We consider that the unique dispersion behavior of dsDNA-NPs is caused by an unidentified but outermost surface-specific interaction between the dsDNA-NPs.

Atomic force microscopy (AFM) is a powerful tool to examine not only the topology but also the interaction between surface-immobilized molecules such as proteins, peptides, and nucleic acids under various conditions. ${ }^{21-23}$ By using AFM probes with a spherical end of defined radius (so-called colloidal probes), the surface force at work between two surfaces can be measured quantitatively. ${ }^{24-26}$ In this study, we have employed the colloidal probe AFM (CP-AFM) technique to examine the interaction between the dsDNA layers. One of the major issues in precisely assessing the effect of subtle structural differences on the surface force using this technique is the structural homogeneity of the surface layers. The dsDNA layer is usually prepared via the hybridization of ssDNA strands with surface-immobilized ssDNA strands. In general, the hybridization efficiency at the solid-liquid interface is lower than that of the solution phase, ${ }^{27,28}$ with some amount of surface-immobilized ssDNA molecules remaining unhybridized, that is, the resultant dsDNA layer has a heterogeneous structure. The process of reducing the residual ssDNA by enhancing the hybridization efficiency on the substrate through the optimization of various factors including the surfaceimmobilized ssDNA density, hybridization temperature, and buffer composition is quite labor-intensive. Nevertheless, a perfect dsDNA layer cannot be guaranteed even after the optimization.

In the present study, we developed a simple alternative method for the preparation of "homogenous" (ssDNA-free) dsDNA layers on a gold surface: the hairpin DNA (hpDNA) anchoring method. First, we applied this method to the dispersion of GNPs and confirmed that the outermost base pairing-specific dispersion behavior was similar to that previously observed for dsDNA-NPs prepared by the surface hybridization procedure. Then, we adopted this method to prepare homogenous dsDNA layers on the surfaces of colloidal probes and gold substrates and measured the force-distance curves between the dsDNA layers using the CP-AFM technique. From the analyses of force-distance curves, we examined the correlation between the dispersion behavior of the dsDNA-NPs and the surface forces at work between the dsDNA layers under corresponding conditions. Finally, we discussed the potential mechanism underlying the outermost base pairing-specificity of the surface forces observed for dsDNA layers.

\section{EXPERIMENTAL SECTION}

2.1. Evaluation of the Colloidal Dispersion Behavior of hpDNA-GNPs. The hpDNA-GNP stock solution was mixed with sodium phosphate buffer $(10 \mathrm{mM}, \mathrm{pH}$ 7.4) containing various concentrations of $\mathrm{NaCl}$. After incubation at $25{ }^{\circ} \mathrm{C}$ for $15 \mathrm{~min}$, the mixture was photographed using a digital camera (CAMEDIA C5050ZOOM; Olympus, Tokyo, Japan). Ultraviolet-visible (UV-vis) absorption spectra measurements were performed on a UV-2550 spectrophotometer equipped with a temperature control unit (Shimadzu, Kyoto, Japan) at $25{ }^{\circ} \mathrm{C}$.

2.2. Force-Distance Curve Measurements between Two dsDNA Layers Using Colloidal Probe AFM. For the forcedistance curve measurements, we used a commercial AFM system equipped with a liquid cell (MFP-3D; Oxford Instruments, Santa Barbara, CA). The spring constant of each colloidal probe was determined by monitoring the thermal fluctuations. The colloidal probe was made to approach the surface until the detection of a 200 $\mathrm{pN}$ repulsion with a speed of $200 \mathrm{~nm} \cdot \mathrm{s}^{-1}$. All force curve measurements were performed in sodium phosphate buffer $(10 \mathrm{mM}$, $\mathrm{pH}$ 7.4) containing various concentrations of $\mathrm{NaCl}(10-1000 \mathrm{mM})$ at room temperature. To avoid the sample-sample variation, the same set of colloidal probe-gold substrate was used with different $\mathrm{NaCl}$ concentrations through the measurements for a specific surface combination, for example, C-DNA-to-C-DNA layers. We measured 20 single-force curves, each at five different positions, for each $\mathrm{NaCl}$ concentration.

2.3. Force-Distance Curve Data Processing. Each single force-distance curve was smoothed with a moving average of 10 sequential data points to reduce noise. We shifted the curves to set the minimum distance data to zero for simplicity. A representative single force-distance curve was picked up for each $\mathrm{NaCl}$ concentration to show the characteristics of the approaching events (Figure 4a,c). A histogram of the maximum attractive force and the corresponding distance was deduced from the single force-distance curves for CDNA layers (Figure $5 \mathrm{a}, \mathrm{b}$ ), whereas forces at a distance of $3.0 \mathrm{~nm}$ were used for M-DNA layers (Figure 5c). The averaged force-distance curves in Figure $4 \mathrm{~b}$,d were produced by binning the data points in 0.1 $\mathrm{nm}$ bins and subsequent averaging of the data in each bin. Because of the scattered jump-in events in Figure 4a, the peak depth and position in Figure $4 \mathrm{~b}$ do not correspond to the average of the maximum attractive force seen in Figure 5a.

\section{RESULTS AND DISCUSSION}

3.1. dsDNA-GNPs Prepared Using the hpDNAAnchoring Method. An outline of the hpDNA-anchoring method is illustrated in Scheme 1. An oligo DNA strand with a

Scheme 1. Outline of the hpDNA-Anchoring Method ${ }^{a}$
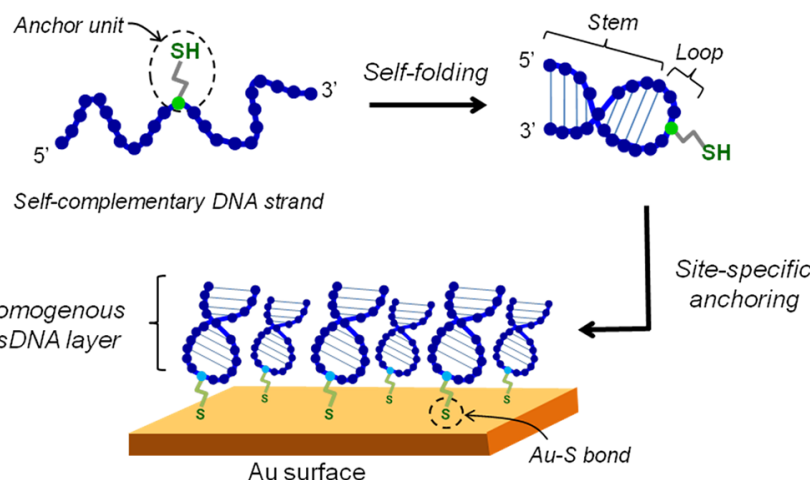

${ }^{a}$ Hairpin structure-folded DNA strands were anchored on the gold surface in a site (loop moiety)-specific manner through $\mathrm{Au}-\mathrm{S}$ bond formation, resulting in the formation of ssDNA-free outer layers consisting solely of stem moieties, that is, homogenous dsDNA layers.

self-complementary sequence spontaneously folds into a stable hairpin structure consisting of stem and loop moieties. We introduced an anchor unit containing a thiol group into the loop moiety to immobilize the hpDNA strands on a gold surface in a site-specific manner with $\mathrm{Au}-\mathrm{S}$ bonding. The surface-immobilized hpDNA strands formed the outer layer consisting solely of stem moieties (dsDNA layer). Our hpDNAanchoring method is more facile and advantageous than the 
surface-hybridization method because this technique guarantees a homogenous (ssDNA-free) surface structure of dsDNA layers on gold surfaces without complicated optimization of hybridization conditions.

The base sequences of 25-mer oligo DNA strands (hpDNA$\mathrm{SH}$ ) used in this study are depicted in Figure 1. The base

(a)

\section{C-DNA-SH}

$5^{\prime}$ - GACAGCTGGTTTXTTACCAGCTGTC- 3'

\begin{tabular}{|c|c|}
\hline $5^{\prime}$-GACAGCTGGT T T & \\
\hline|||||||| $\mid$ & \\
\hline $3^{\prime}$ - CTGTCGACCA T T & $\mathrm{X}=\mathrm{dTC}_{6} \mathrm{SH}$ \\
\hline
\end{tabular}

M-DNA-SH

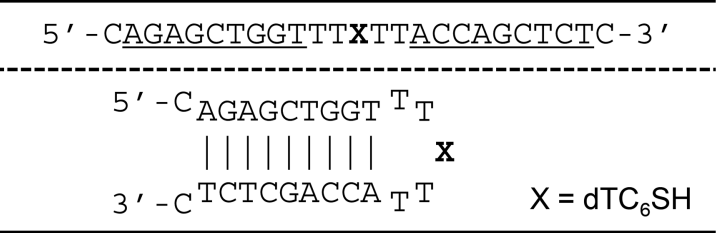

(b)

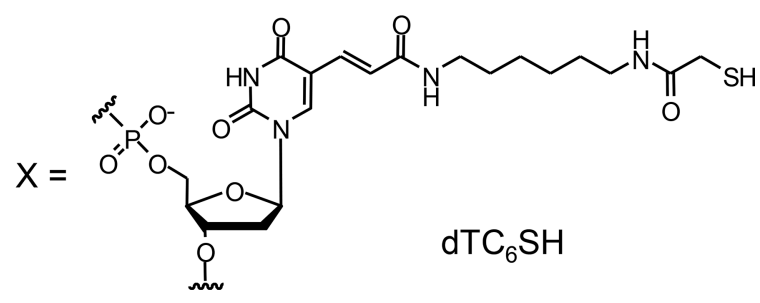

Figure 1. (a) Base sequences and folded structures of hpDNA-SH. Self-complementary regions in hpDNA-SH sequences are shown as underlined text. Upon folding, each hpDNA-SH forms a 5 mer loop of $\mathrm{T}$ with a hairpin stem. (b) Chemical structure of the anchor unit (X: $\mathrm{dT}-\mathrm{SH}$ ). A thiol group acts as an anchor for the gold surface.

sequences of hpDNA-SH with a complementary/mismatched terminal base pair (C-DNA-SH, M-DNA-SH) were designed to fold into a hairpin structure using an mfold DNA Web-based program. ${ }^{29}$ Upon folding, each oligo DNA forms the 5-mer loop of T (T5 loop) with a hairpin stem. C-DNA-SH has a complementary stem terminal (G-C pair), and M-DNA-SH has a single-base mismatch at the stem terminal ( $\mathrm{C}-\mathrm{C}$ pair). The stability of these hairpin structures was guaranteed by melting temperature $\left(T_{\mathrm{m}}\right)$ measurements (Figure $\mathrm{S} 1$ ). For anchoring of hpDNA strands on GNPs, a thiol group was introduced through an alkyl spacer unit (X: dT-SH; Figure 1) at the fifth position of the thymine base of the T5 loop center. Through Au-S bonding, the hpDNA-SH strands were sitespecifically anchored on the gold surface, forming a homogenous dsDNA layer as illustrated in Scheme 1. Details of the synthesis and characterizations of hpDNA-SH are described in the Supporting Information.

Two types of hpDNA-functionalized GNPs (hydrodynamic diameter of GNP core $=41.0 \mathrm{~nm}$ ), that is, C-DNA-GNPs and M-DNA-GNPs, were prepared using the hpDNA-anchoring method. Both dispersions were stable in the presence of $50 \mathrm{mM}$ $\mathrm{NaCl}$ and showed a clear red color. The hydrodynamic diameters of C-DNA-GNPs and M-DNA-GNPs estimated using dynamic light scattering measurements were 46.4 and $45.6 \mathrm{~nm}$, respectively. The site-specific immobilization of the hpDNA-SH strands on the GNPs was confirmed by the incubation of bare GNPs with anchor-free hpDNA (C-hpDNA, M-hpDNA) and subsequent addition of $50 \mathrm{mM} \mathrm{NaCl}$, which resulted in the immediate aggregation of the GNPs (Figure S5). The immediate aggregation revealed, in turn, that the hpDNA$\mathrm{SH}$ strands were anchored specifically on the GNPs with $\mathrm{Au}-\mathrm{S}$ bonding. Moreover, the anchoring of hpDNA-SH strands via $\mathrm{Au}-\mathrm{S}$ bonding guarantees the formation of a layer with an exposed stem terminal (dsDNA layer) as the outermost structure, as we expected in Scheme 1.

The number of hpDNA-SH strands immobilized on the GNPs was evaluated using the dithiothreitol (DTT) displacement assay. $^{30}$ The average number of C-DNA-SH strands immobilized on the GNP surface was $309 \pm 17$ strands per particle (surface area of C-DNA-SH of $16.9 \pm 0.8 \mathrm{~nm}^{2}$ ), whereas that of M-DNA-SH strands was $313 \pm 4$ strands per particle (surface area of M-DNA-SH of $16.8 \pm 0.2 \mathrm{~nm}^{2}$ ). The difference between the two was sufficiently small, indicating that the stem terminal structure of hpDNA-SH had almost no effect on the efficiency of immobilization on the gold surface. The above-mentioned results clearly demonstrate that the hpDNA-anchoring method is a powerful means of preparing a dsDNA layer on gold surfaces with an ssDNA-free, stem terminal base-pair-independent, and well-controlled DNA strand configuration.

3.2. Colloidal Dispersion Behavior of hpDNA-Functionalized GNPs. The colloidal dispersion behavior of the hpDNA-functionalized GNPs exhibited a strong dependence on their outermost base pairs. As shown in Figure 2a, the dispersion of C-DNA-GNPs showed a distinctive color change from red to light purple when the concentration of $\mathrm{NaCl}$ in the dispersion was above $250 \mathrm{mM}$, indicating that the C-DNAGNPs aggregated in a non-cross-linking manner. The UV-vis absorption spectra also qualitatively supported the $\mathrm{NaCl}$ induced non-cross-linking aggregation of C-DNA-GNPs (Figure 2b,c). By contrast, the M-DNA-GNPs showed an extremely high colloidal dispersion stability with no visible change in color (Figure 3a) and no significant change in UVvis absorption spectra over the entire $\mathrm{NaCl}$ concentration range examined up to $1 \mathrm{M}$ (Figure $3 \mathrm{~b}, \mathrm{c}$ ). It should be emphasized that the difference between C-DNA-GNPs and M-DNAGNPs is only the outermost base pair (complementary or mismatched) because the other factors such as remaining unhybridized ssDNAs or unfavorably aligned DNAs were completely excluded with the hpDNA-anchoring method. The result, therefore, strongly suggests that the terminal difference of the surface-tethered dsDNA evokes a large deviation in the physicochemical properties of the hpDNA-functionalized GNP surface, leading to the obvious difference in the colloidal dispersion behavior observed. In addition, the clear contrast in the colloidal dispersion behavior observed for hpDNAfunctionalized GNPs is consistent with our previous observations on dsDNA-grafted NPs prepared using surface hybridization, ${ }^{15-20}$ where unhybridized ssDNAs might remain on the surface of the NPs.

One possible explanation for the above-described difference in the dispersion behavior of hpDNA-functionalized GNPs is that these particles may have a different surface charge. The 
(a)

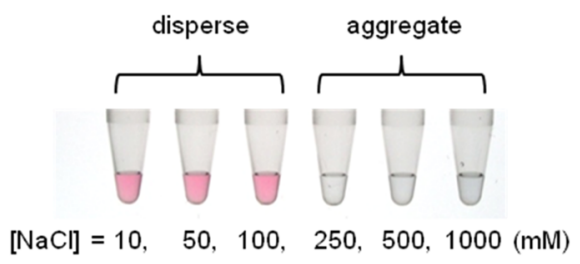

(b)

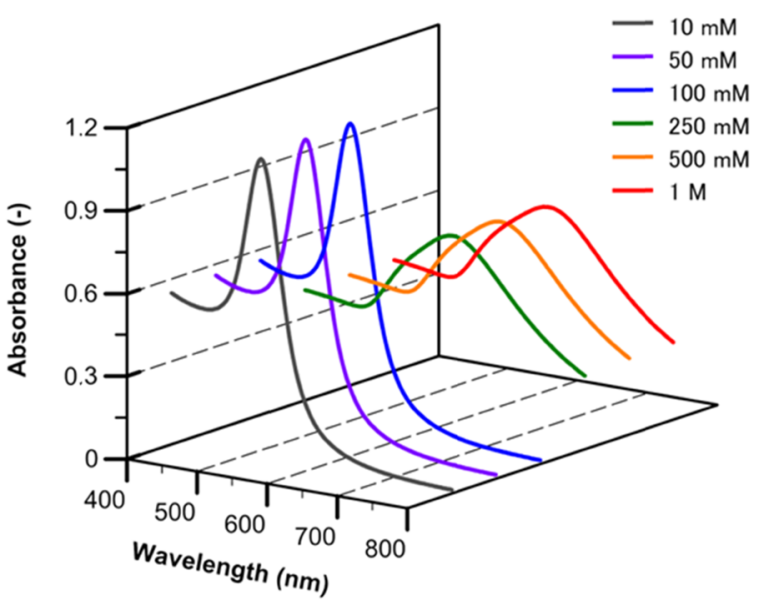

(c)

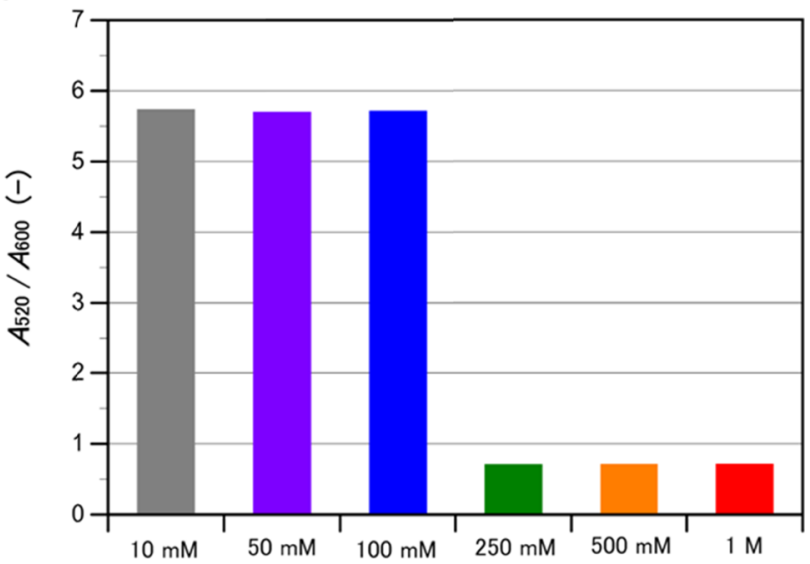

Figure 2. Photographs (a), UV-vis spectra (b), and the ratio of absorption observed at 520 and $600 \mathrm{~nm}\left(A_{520} / A_{600}\right)$ (c) for the CDNA-GNP dispersions in sodium phosphate buffer $(10 \mathrm{mM}, \mathrm{pH} 7.4)$ containing various concentrations of $\mathrm{NaCl}$ at $25{ }^{\circ} \mathrm{C}$. Above $250 \mathrm{mM}$ $\mathrm{NaCl}$, the C-DNA-GNP dispersion showed a distinctive change in color and UV-vis spectrum, revealing the aggregation.

surfaces charge for C-DNA-GNPs and M-DNA-GNPs were evaluated using zeta potential measurements at various $\mathrm{NaCl}$ concentrations $(10,50,100$, and $250 \mathrm{mM})$. With an increase in $\mathrm{NaCl}$ concentration, the absolute value of the zeta potential decreased to $-45.1 \pm 2.5(-40.5 \pm 0.7) \mathrm{mV}$ for $10 \mathrm{mM}$ and $-24.4 \pm 1.1(-24.6 \pm 1.3) \mathrm{mV}$ for $250 \mathrm{mM}$ for C-DNAGNPs (M-DNA-GNPs), as shown in Figure S6, but no significant difference was observed between C-DNA-GNPs and M-DNA-GNPs. Therefore, we concluded that an electrostatic factor is not responsible for the clear contrast in the colloidal dispersion behavior observed for C-DNA-GNPs and M-DNA-GNPs.

3.3. Interaction between dsDNA Layers Measured Using CP-AFM. To examine how the outermost base pairs (a)

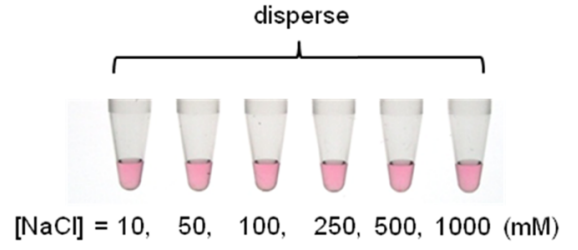

(b)

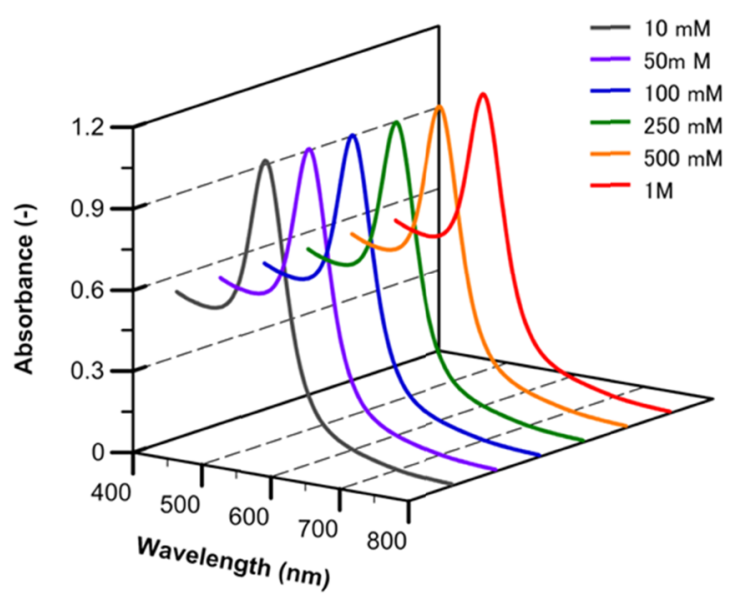

(c)

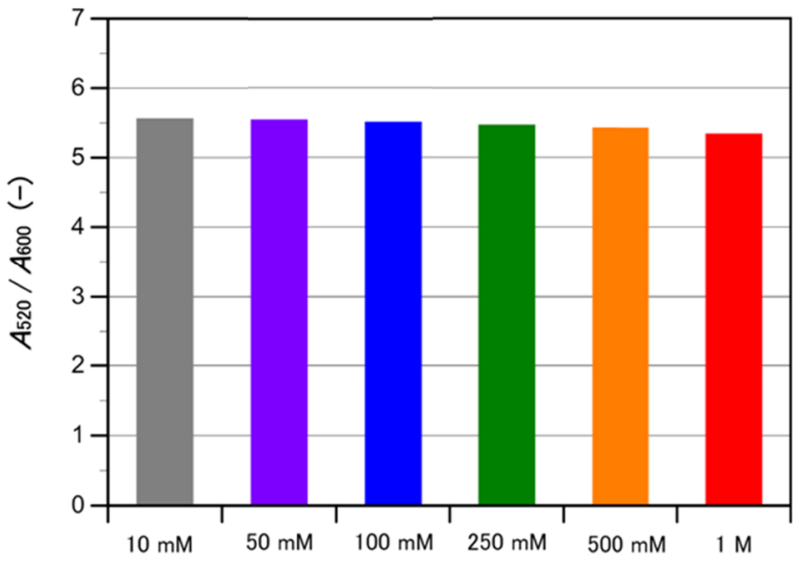

Figure 3. Photographs (a), UV-vis spectra (b), and the ratio of absorption observed at 520 and $600 \mathrm{~nm}\left(A_{520} / A_{600}\right)$ (c) for the MDNA-GNP dispersions in sodium phosphate buffer $(10 \mathrm{mM}, \mathrm{pH} 7.4)$ containing various concentrations of $\mathrm{NaCl}$ at $25^{\circ} \mathrm{C}$. Unlike C-DNAGNP dispersion, the M-DNA-GNP dispersion showed no significant change in either the color or the UV-vis spectrum even in high $\mathrm{NaCl}$ concentrations.

affect the surface force between the dsDNA layers, forcedistance curve measurements were taken using the CP-AFM technique by using hpDNA-functionalized colloidal probes and substrates. The force-distance dependence between the dsDNA layers was assessed in sodium phosphate buffer (10 $\mathrm{mM}, \mathrm{pH} 7.4)$ containing various concentrations of $\mathrm{NaCl}$ (10$1000 \mathrm{mM}$ ).

3.3.1. Force-Distance Dependence between C-DNA Layers. Representative single force-distance curves observed for approaching C-DNA layers at various $\mathrm{NaCl}$ concentrations up to a force of $200 \mathrm{pN}$ are shown in Figure 4a, and their averaged ones (more than 100 curves) are shown in Figure $4 \mathrm{~b}$. The characteristic attractive force with a jump-in event was clearly observed for $\mathrm{NaCl}$ concentrations above $250 \mathrm{mM}$ in Figure $4 \mathrm{a}$, and this force increased with the $\mathrm{NaCl}$ concentration. 
(a)
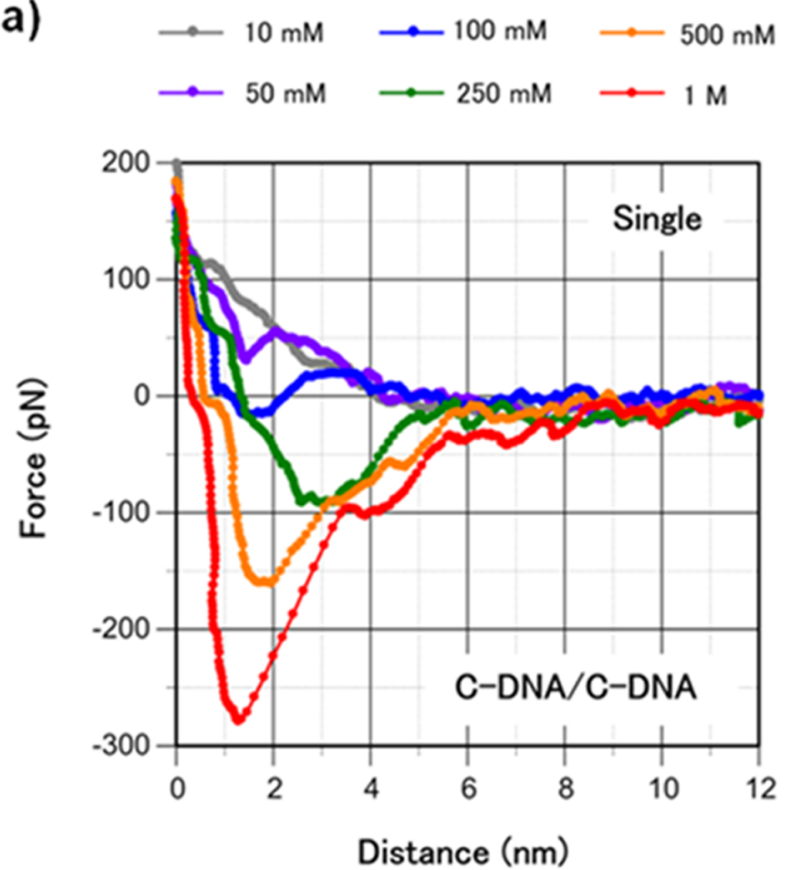

(c)

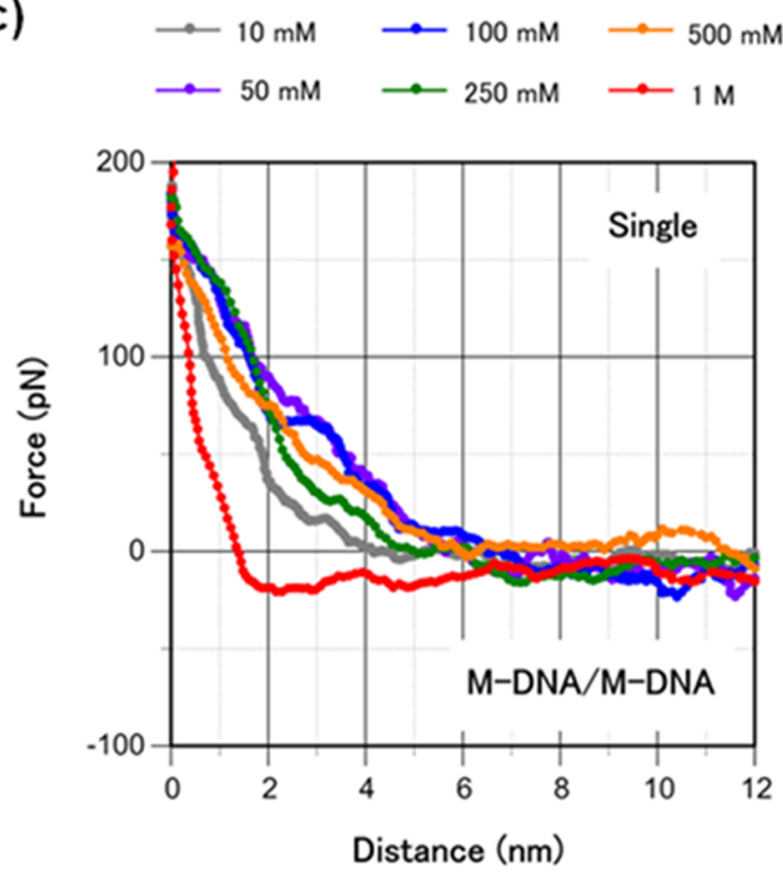

(b)
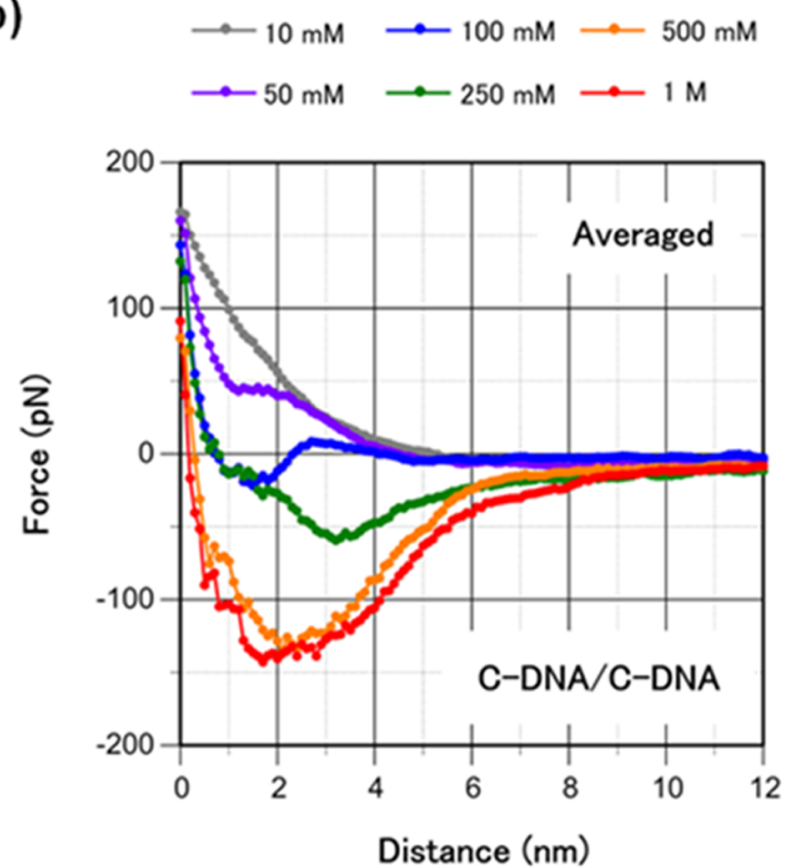

(d)

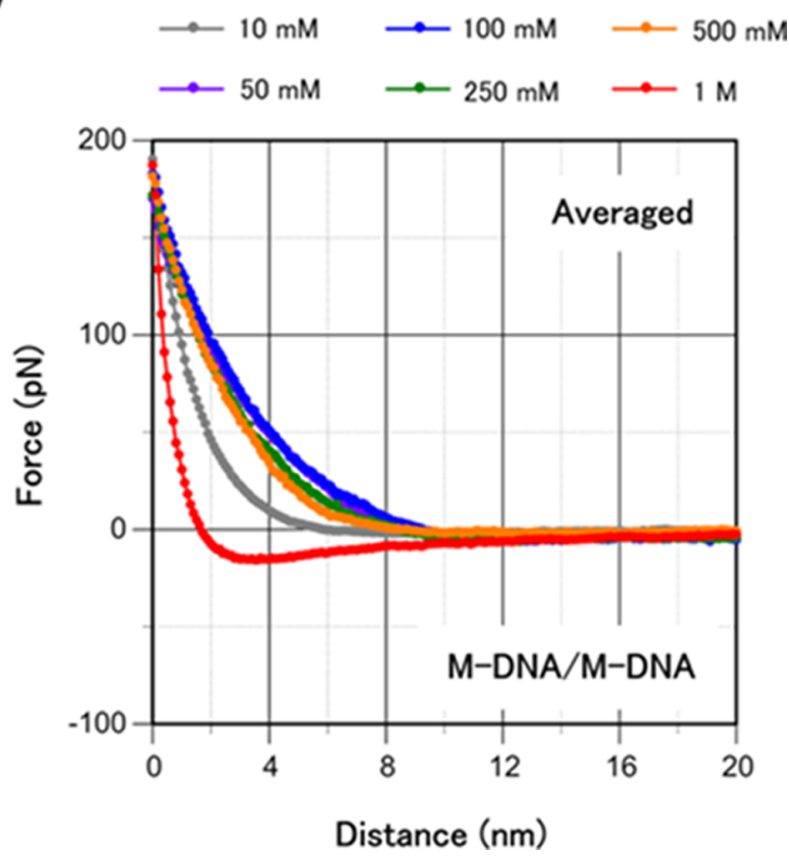

Figure 4. Representative single force-distance curves (a) and averaged curves (b) obtained for the approaching C-DNA layers in sodium phosphate buffer (10 mM, pH 7.4) containing various concentrations of $\mathrm{NaCl}$. Straight slopes in the curves in (a) correspond to jump-in events, that is, the probe was pulled to the surface with a strong attractive force. The deviation in the peak shape between single and averaged curves was due to the scattered distribution of maximum attraction distances, as plotted in Figure 5 (b). Representative single force-distance curves (c) and averaged curves (d) obtained for approaching M-DNA layers.

A small dip related to the jump-in event was also observed on the curves for $\mathrm{NaCl}$ concentrations lower than $100 \mathrm{mM}$, although the surface force was mostly repulsive. As will be mentioned later, the jump-in event takes place in a stochastic manner, resulting in a scattered depth (force strength) and position (distance) of the valley on the curves. The dependence of the force-distance curve on the $\mathrm{NaCl}$ concentration can be discussed more qualitatively based on the averaged curves in Figure $4 b$, where the increase in the attractive interaction with the $\mathrm{NaCl}$ concentration is also observed and the attractive force becomes obvious at a distance of approximately $8 \mathrm{~nm}$.

One important point that should be noted from the series of force-distance curves is that the increase in the $\mathrm{NaCl}$ concentration facilitates the attractive interaction between the C-DNA layers, rather than reducing the repulsive interaction. When approaching the C-DNA layers at a $\mathrm{NaCl}$ concentration above $250 \mathrm{mM}$, the attractive interaction begins at a distance of $8 \mathrm{~nm}$ (Figure 4b) and leads to a jump-in event at a distance of 
1-5 nm (Figure 4a). This means that the attractive force observed here cannot be assigned to van der Waals force, which usually shows no significant dependence on the salt concentration. $^{31}$ On the basis of our observation that the attractive force was strongly dependent on the $\mathrm{NaCl}$ concentration, we consider that the hydrophobic interaction between the C-DNA layers facilitated by $\mathrm{NaCl}$ is the most probable origin of the attraction. A further discussion of the hydrophobic interaction, that is, the molecular mechanism of the attraction between the C-DNA layers facilitated by $\mathrm{NaCl}$, will be made in a later section.

Figure 5 shows the histograms for (a) the maximum attraction force (the lowest force value on the curve) observed above $100 \mathrm{mM} \mathrm{NaCl}$ and (b) the corresponding distance where the maximum attraction was achieved. Upon increasing the $\mathrm{NaCl}$ concentration from $100 \mathrm{mM}$ to $1 \mathrm{M}$, the distribution of the maximum attraction force became broad. The distribution of the corresponding distance was similarly scattered within this $\mathrm{NaCl}$ concentration range. The broad distribution of the attraction strength and distance indicates that the attractive interaction between the C-DNA layers varies from event to event, that is, the jump-in event takes place in a stochastic manner.

3.3.2. Force-Distance Dependence between M-DNA Layers. The force-distance curves observed for the approaching M-DNA layers are shown in Figure $4 c, d$ for single and averaged curves (more than 100 curves). Figure 5c shows the histogram of the force observed at a distance of $3 \mathrm{~nm}$, revealing a higher reproducibility of the force-distance curves in Figure $4 c$, compared with that for C-DNA layers (Figure 4a). Unlike in the case of the C-DNA layers, the force existing between the MDNA layers was simply repulsive with an exponential-type decay with distance, except at a $\mathrm{NaCl}$ concentration of $1 \mathrm{M}$. Notably, the repulsion force starts as it approaches to within a distance of approximately $8 \mathrm{~nm}$, and the force-distance curves showed no significant dependence on the $\mathrm{NaCl}$ concentration within the range of $50-500 \mathrm{mM}$ (Figure $4 \mathrm{~d}$ ). The observed distance of $8 \mathrm{~nm}$ is far beyond the distance range of the electrostatic force, which has been evaluated as $1.2 \mathrm{~nm}$ for a $\mathrm{NaCl}$ concentration of $10 \mathrm{mM}$ in sodium phosphate buffer (10 $\mathrm{mM}, \mathrm{pH}$ 7.4), deduced as the Debye length. ${ }^{32}$ The small deviation observed for $10 \mathrm{mM} \mathrm{NaCl}$ was reproducible, but the reason for the deviation is not clear at the moment. Only at 1 $\mathrm{M}$ of $\mathrm{NaCl}$, we observed a weak attractive interaction on the single force-distance curves, but we rarely observed an attractive force higher than $50 \mathrm{pN}$ (Figure S7). On the averaged curve, we observed an attraction of approximately 20 $\mathrm{pN}$ at $1 \mathrm{M} \mathrm{NaCl}$ (Figure 4d), which was far below that for the C-DNA layers, revealing that the attractive interaction present between the M-DNA layers was not significant even at higher $\mathrm{NaCl}$ concentrations. Nevertheless, the existence of a weak attractive interaction between the M-DNA layers was revealed by the force-distance curve measurements, and such an interaction cannot be deduced from the dispersion behavior of M-DNA-GNPs.

The onset of the repulsive force observed on the averaged curves in Figure 4d was approximately $8 \mathrm{~nm}$ for $50-500 \mathrm{mM}$ $\mathrm{NaCl}$, whereas it was approximately $1 \mathrm{~nm}$ for $1 \mathrm{M} \mathrm{NaCl}$. At the same time, the repulsive force increased slowly for $50-500 \mathrm{mM}$ $\mathrm{NaCl}$, compared with the steep increase observed for $1 \mathrm{M}$ $\mathrm{NaCl}$. It seems reasonable to attribute this steep increase to the soft and direct contact of the M-DNA layers. The differences in the force-distance curves suggested that the properties of the (a)

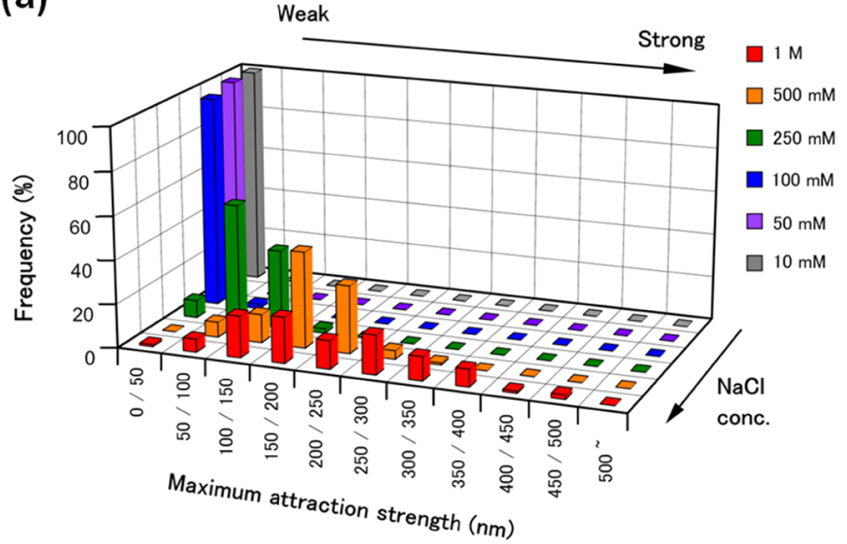

(b)

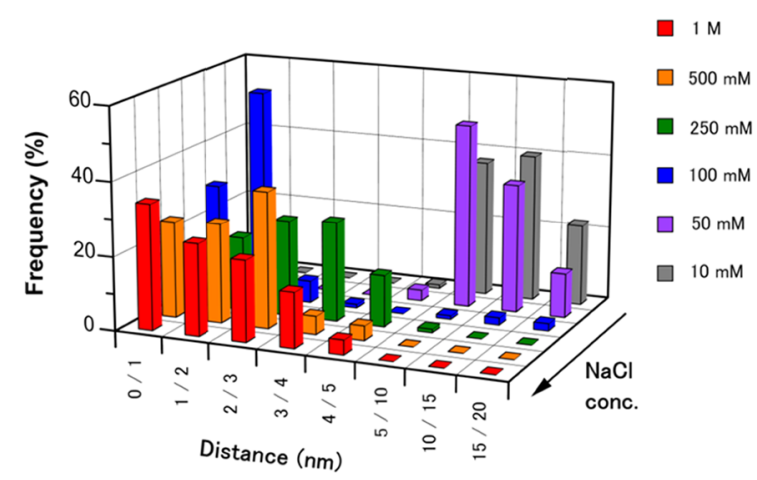

(c)

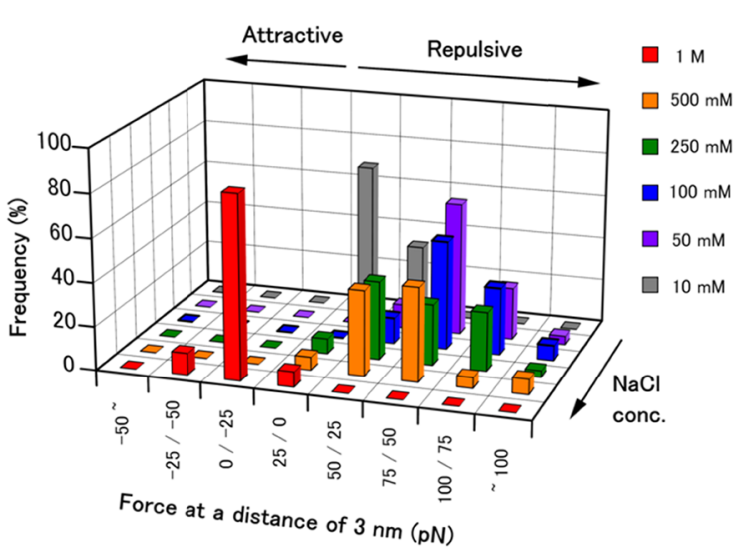

Figure 5. Histograms for (a) the minimum force value (maximum attraction force) for C-DNA layers within a distance of $20 \mathrm{~nm}$ and (b) the distance of maximum attraction point for C-DNA layers. The average maximum attraction forces were 18, 21, 45, 90, 181, and 224 $\mathrm{pN}$ for $\mathrm{NaCl}$ concentrations of 10,50,100, 250, 500, and $1000 \mathrm{mM}$, respectively, and forces less than $50 \mathrm{pN}$ could be attributed to a fluctuation error, as evidenced by the distance distribution beyond 10 $\mathrm{nm}$ in (b). (c) Force observed at a distance of $3 \mathrm{~nm}$ for M-DNA layers. The standard deviation for the distribution was less than $25 \mathrm{pN}$ for all $\mathrm{NaCl}$ concentrations, which could be attributed to measurement fluctuations.

M-DNA layers and the surrounding solvent environments were drastically changed at a $\mathrm{NaCl}$ concentration of $1 \mathrm{M}$.

3.4. Mechanism of the Outermost Base Pair-Specific Surface Forces between dsDNA Layers. The average values of the maximum attraction force for the C-DNA layers and the force observed at a distance of $3 \mathrm{~nm}$ for the M-DNA-layers were plotted as a function of the $\mathrm{NaCl}$ concentration, together with the absorption ratio $\left(A_{520} / A_{600}\right)$ of the corresponding 
hpDNA-functionalized GNP dispersions (Figure 6). The $\mathrm{NaCl}$ concentration dependence of each force agrees well with that of

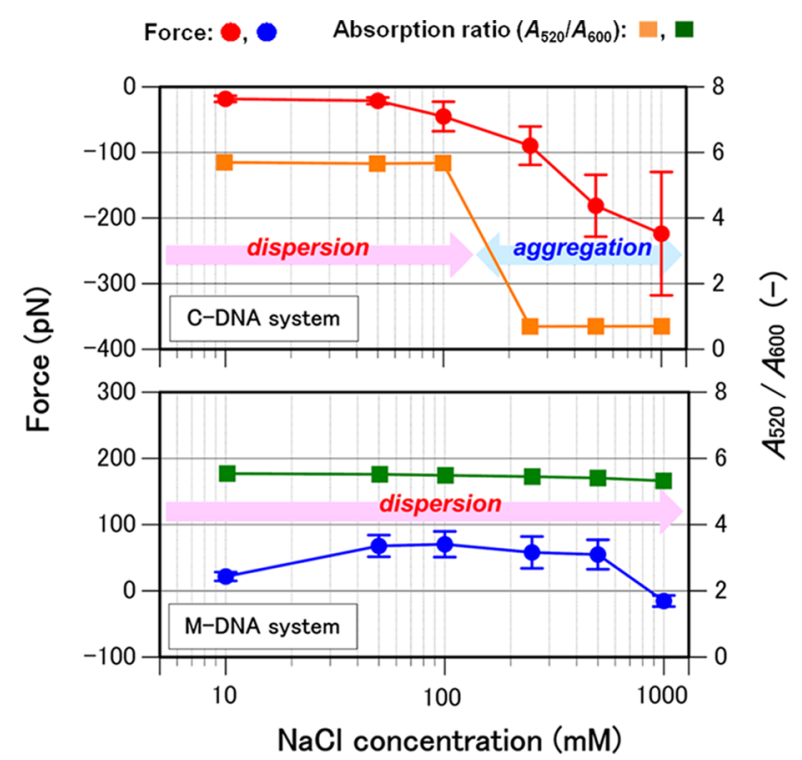

Figure 6. Correlation between the dispersion behavior of hpDNAGNPs and the surface force of dsDNA layers at various $\mathrm{NaCl}$ concentrations. The dispersion behavior of hpDNA-GNPs as expressed by the absorption ratio $\left(A_{520} / A_{600}\right)$ was plotted as a function of $\mathrm{NaCl}$ concentration. The maximum attraction force observed within a distance of $20 \mathrm{~nm}$ was plotted for C-DNA layers, whereas the force observed at a distance of $3 \mathrm{~nm}$ was plotted for MDNA layers.

the dispersion/aggregation behavior of dsDNA-functionalized GNPs, showing the significant correlation between the surface force and the dispersion behavior. Aggregation of hpDNAfunctionalized GNPs occurred $\left(A_{520} / A_{600}\right.$ value was decreased $)$ when the attractive force between the corresponding dsDNA layers reached above $89 \mathrm{pN}\left(9.9 \mathrm{~N} \cdot \mathrm{m}^{-1}\right)$. The weak attractive force observed for the C-DNA layers at $100 \mathrm{mM} \mathrm{NaCl}$ (ca. 45 $\mathrm{pN}, 5.0 \mathrm{~N} \cdot \mathrm{m}^{-1}$ ) and the M-DNA layers at $1 \mathrm{M} \mathrm{NaCl}$ (ca. 15 $\mathrm{pN}, 1.7 \mathrm{~N} \cdot \mathrm{m}^{-1}$ ) had no significant effect on the dispersion state of hpDNA-functionalized GNPs, suggesting that the strength of the attraction force was too weak to maintain contact between GNPs against their Brownian motion.

Notably, a large attractive force was observed only for CDNA layers with higher $\mathrm{NaCl}$ concentrations. This was attributed to a terminal-specific attraction between complementary dsDNA terminals facilitated by $\mathrm{NaCl}$. In general, an increase in the salt concentration reduces the water activity, leading to a dehydration of polyelectrolytes including DNA. ${ }^{33,34}$ We observed a characteristic change in the circular dichroism (CD) spectra of hpDNA solutions with an increase in the $\mathrm{NaCl}$ concentration from $10 \mathrm{mM}$ to $1 \mathrm{M}$ (Figure S8), which could be assigned to the gradual conformational change of the stem moiety (B-form to $\mathrm{C}$-form) by reducing the degree of hydration. Correlating the strong dependence of the attractive interaction between the C-DNA layers on the $\mathrm{NaCl}$ concentration (Figure 6) with the reduction in the degree of hydration suggested by the $\mathrm{CD}$ spectra, we conclude that a hydrophobic interaction is the most probable origin of the attraction. We consider that the terminal-specific hydrophobic interaction is the end-to-end attraction of dsDNA strands with complementary terminals, the so-called blunt-end stack- ing. ${ }^{35-44}$ This consideration was experimentally supported by the force-distance curve measurements taken for the heterogenic surface combination of an M-DNA layer and a C-DNA layer. In this measurement, no significant attraction appears even at $1 \mathrm{M} \mathrm{NaCl}$ (Figure S9), revealing that the attraction functions selectively between dsDNA strands with complementary terminals, that is, the $\mathrm{NaCl}$-facilitated attractive interaction takes place only between the C-DNA layers.

The appearance of the blunt-end stacking between the DNA strands has typically been reported at locally concentrated DNAs such as the DNA tile, ${ }^{36}$ DNA bundle, ${ }^{37}$ DNA liquid crystal, $^{38}$ and DNA origami. ${ }^{39-42}$ Pollack and co-workers reported that divalent salts such as $\mathrm{MgCl}_{2}$ (above $20 \mathrm{mM}$ ) facilitate the blunt-end stacking between complementary dsDNA strands even in a semidilute solution $(\sim 10 \mathrm{mM}){ }^{43,44}$ They also revealed that monovalent salts including $\mathrm{NaCl}$ have no ability to promote the blunt-end stacking even at high concentrations $(\sim 650 \mathrm{mM}) .^{43}$ By contrast, our present results revealed that the concentration of $\mathrm{NaCl}$ above $250 \mathrm{mM}$ promotes blunt-end attraction between dsDNA layers with complementary terminals (Figure $4 \mathrm{a}, \mathrm{b}$ ). This might be due to the surface-anchoring of the dsDNA strands. The diffusional motion of the dsDNA strands is strongly restricted by anchoring to the gold surface, and the resultant configuration promotes terminal-terminal interaction between the dsDNA layers on approaching. In addition, the reduction in the number of water molecules surrounding dsDNA with an increase in $\mathrm{NaCl}$ concentration enables dsDNA terminals to approach more closely. The terminal closure promotes attractive interactions between complementary dsDNA terminals through the blunt-end stacking.

On the other hand, dsDNA strands with mismatched terminals cannot attract each other even by approaching closely because of their lack of hydrophobic terminal base pairs. In addition, the number of potential binding sites of water molecules to the unpairing terminal nucleobases is larger than that of pairing ones (Figure S10). This leads to the persistence of tightly bound water molecules surrounding the mismatched dsDNA terminals even at a higher $\mathrm{NaCl}$ concentration, resulting in the persistent repulsion force between the $M$ DNA layers observed in Figure 6 up to a salt concentration of $500 \mathrm{mM}$. The working distance of the repulsion observed in Figure $4 c, d$ was larger than twice the thickness of the dsDNA layer (estimated to be approximately $7 \mathrm{~nm}$ ), supporting the notion that environmental factors such as water configuration are important for the repulsion interaction of the mismatched dsDNA terminals. When the number of water molecules binding to the terminal moiety is reduced at $1 \mathrm{M} \mathrm{NaCl}$, the $\mathrm{M}$ DNA layers can approach more closely together, but the attractive interaction is too weak to form a stable adhesion (colloidal aggregation), as indicated by the significantly small attraction observed in Figure 6.

\section{CONCLUSIONS}

We explored the mechanism underlying the outermost base pairing dependence of the dispersion behavior of dsDNA-NPs. Site-specific immobilization of the hpDNA strands enabled us to prepare homogenous dsDNA layers on gold surfaces with complementary or mismatched outermost base pairs. The force-distance curve analyses between the dsDNA layers using the CP-AFM technique showed that the outermost complementary (mismatched) dsDNA layers attract (repulse) each other at a higher $\mathrm{NaCl}$ concentration. These results agree well 
with the outermost base pairing dependence of the colloidal dispersion behavior of dsDNA-NPs, indicating that the outermost base-pairing-specific interaction between the dsDNA-NPs dominates their dispersion behavior at a higher $\mathrm{NaCl}$ concentration. We concluded that the $\mathrm{NaCl}$-facilitated end-to-end hydrophobic interaction (blunt-end stacking) between complementary dsDNA terminals caused the attraction between the C-DNA layers, whereas the repulsive interaction observed between the M-DNA layers was mainly attributable to a repulsive hydration force. Although the number of dsDNA pairs involved in the surface force is not clear at this moment, this is the first direct observation of the terminal-specific interaction between dsDNA layers in terms of force, to the best of our knowledge. We believe that our findings will contribute not only to designing the dsDNA interface-based molecular systems ${ }^{45-47}$ including single-nucleotide polymorphism (SNP) genotyping devices ${ }^{48}$ but also to understanding the molecular basis of DNA-DNA interactions in biology. In fact, the concentration of monovalent cations (sodium and potassium ions) in the nucleoplasm is reported to be approximately $500 \mathrm{mM}^{42,49}$ Further studies along these lines are currently under way.

\section{ASSOCIATED CONTENT}

\section{S Supporting Information}

The Supporting Information is available free of charge on the ACS Publications website at DOI: 10.1021/acs.langmuir.6b03470.

Experimental details, melting curves, and melting temperature of hpDNAs, synthesis of thiol groupincorporated hairpin DNAs (hpDNA-SHs), colloidal dispersion stability of hpDNA/GNP mixtures, zeta potentials of C-DNA-GNPs and M-DNA-GNPs at various $\mathrm{NaCl}$ concentrations, histogram of minimum force value observed on approaching M-DNA layers, CD spectra of hpDNA, force-distance dependence observed for the approaching M-DNA layer and C-DNA layer, and the potential binding site of water molecules to the pairing/unpairing terminal base pairs (PDF)

\section{AUTHOR INFORMATION}

\section{Corresponding Authors}

*E-mail: nkanayama@shinshu-u.ac.jp (N.K.).

*E-mail: hayashi@echem.titech.ac.jp (T.H.).

ORCID

Naoki Kanayama: 0000-0001-8592-2757

Notes

The authors declare no competing financial interest.

\section{ACKNOWLEDGMENTS}

This work was financially supported by JSPS KAKENHI Grant Numbers JP25220204 (N.K. and M.M.), JP26410232 (N.K.) and JP26282118 (T.H.). This work was also supported by a grant for the Molecular-Systems Research provided by RIKEN. The authors are grateful to Dr. Takehiro Suzuki from the Collaboration Promotion Unit of the Global Research Cluster at RIKEN for MALDI-TOF mass spectroscopy measurements.

\section{REFERENCES}

(1) Petrovykh, D. Y.; Kimura-Suda, H.; Whitman, L. J.; Tarlov, M. J. Quantitative Analysis and Characterization of DNA Immobilized on Gold. J. Am. Chem. Soc. 2003, 125, 5219-5226.
(2) Shen, G.; Tercero, N.; Gaspar, M. A.; Varughese, B.; Shepard, K.; Levicky, R. Charging Behavior of Single-Stranded DNA Polyelectrolyte Brushes. J. Am. Chem. Soc. 2006, 128, 8427-8433.

(3) White, R. J.; Phares, N.; Lubin, A. A.; Xiao, Y.; Plaxco, K. W. Optimization of Electrochemical Aptamer-Based Sensors via Optimization of Probe Packing Density and Surface Chemistry. Langmuir 2008, 24, 10513-10518.

(4) Walter, S. R.; Geiger, F. M. DNA on Stage: Showcasing Oligonucleotides at Surfaces and Interfaces with Second Harmonic and Vibrational Sum Frequency Generation. J. Phys. Chem. Lett. 2010, $1,9-15$.

(5) Watkins, H. M.; Simon, A. J.; Ricci, F.; Plaxco, K. W. Effects of Crowding on the Stability of a Surface-Tethered Biopolymer: An Experimental Study of Folding in a Highly Crowded Regime. J. Am. Chem. Soc. 2014, 136, 8923-8927.

(6) Akamatsu, K.; Kimura, M.; Shibata, Y.; Nakano, S.-i.; Miyoshi, D.; Nawafune, H.; Sugimoto, N. A DNA Duplex with Extlemely Enhanced Thermal Stability Based on Controlled Immobilization on Gold Nanoparticles. Nano Lett. 2006, 6, 491-495.

(7) Long, H.; Kudlay, A.; Schatz, G. C. Molecular Dynamics Studies of Ion Distributions for DNA Duplexes and DNA Clusters: Salt Effects and Connection to DNA Melting. J. Phys. Chem. B 2006, 110, 29182926.

(8) Seferos, D. S.; Prigodich, A. E.; Giljohann, D. A.; Patel, P. C.; Mirkin, C. A. Polyvalent DNA Nanoparticle Conjugates Stabilize Nucleic Acids. Nano Lett. 2009, 9, 308-311.

(9) Lei, Q.-1.; Ren, C.-1.; Su, X.-h.; Ma, Y.-q. Crowding-Induced Cooperativity in DNA Surface Hybridization. Sci. Rep. 2015, 5, 9217.

(10) Cutler, J. I.; Auyeung, E.; Mirkin, C. A. Spherical Nucleic Acids. J. Am. Chem. Soc. 2012, 134, 1376-1391.

(11) Elghanian, R.; Storhoff, J. J.; Mucic, R. C.; Letsinger, R. L.; Mirkin, C. A. Selective Colorimetric Detection of Polynucleotides Based on the Distance-Dependent Optical Properties of Gold Nanoparticles. Science 1997, 277, 1078-1081.

(12) Geerts, N.; Eiser, E. DNA-Functionalized Colloids: Physical Properties and Applications. Soft Matter 2010, 6, 4647-4660.

(13) Taton, T. A.; Mucic, R. C.; Mirkin, C. A.; Letsinger, R. L. The DNA-Mediated Formation of Suplamolecular Mono- and Mulilayered Nanoparticle Structures. J. Am. Chem. Soc. 2000, 122, 6305-6306.

(14) Jin, R.; Wu, G.; Li, Z.; Mirkin, C. A.; Schatz, G. C. What Controls the Melting Properties of DNA-Linked Gold Nanoparticle Assemblies? J. Am. Chem. Soc. 2003, 125, 1643-1654.

(15) Sato, K.; Hosokawa, K.; Maeda, M. Rapid Aggregation of Gold Nanoparticles Induced by Non-Cross-Linking DNA Hybridization. J. Am. Chem. Soc. 2003, 125, 8102-8103.

(16) Sato, K.; Sawayanagi, M.; Hosokawa, K.; Maeda, M. Single-Base Mutation Detection Using Neutravidin-Modified Polystyrene Nanoparticle Aggregation. Anal. Sci. 2004, 20, 893-894.

(17) Isoda, K.; Kanayama, N.; Fujita, M.; Takarada, T.; Maeda, M. DNA Terminal Mismatch-Induced Stabilization of Polymer Micelles from RAFT-Generated Poly( $N$-isopropylacrylamide)-DNA Block Copolymers. Chem.-Asian J. 2013, 8, 3079-3084.

(18) Sato, K.; Onoguchi, M.; Sato, Y.; Hosokawa, K.; Maeda, M. Non-Cross-Linking Gold Nanoparticle Aggregation for Sensitive Detection of Single-Nucleotide Polymorphisms: Optimization of the Particle Diameter. Anal. Biochem. 2006, 350, 162-164.

(19) Fujita, M.; Katafuchi, Y.; Ito, K.; Kanayama, N.; Takarada, T.; Maeda, M. Structural Study on Gold Nanoparticle Functionalized with DNA and Its Non-Cross-Linking Aggregation. J. Colloid Interface Sci. 2012, 368, 629-635.

(20) Wang, G.; Akiyama, Y.; Takarada, T.; Maeda, M. Rapid NonCrosslinking Aggregation of DNA-Functionalized Gold Nanorods and Nanotriangles for Colorimetric Single-Nucleotide Discrimination. Chem.-Eur. J. 2016, 22, 258-263.

(21) Butt, H.-J.; Cappella, B.; Kappl, M. Force Measurements with the Atomic Force Microscope: Technique, Interpretation and Applications. Surf. Sci. Rep. 2005, 59, 1-152.

(22) Scanning Probe Microscopies Beyond Imaging; Samorì, P., Ed.; Wiley-VCH: Weinheim, 2006. 
(23) Mochizuki, M.; Oguchi, M.; Kim, S.-O.; Jackman, J. A.; Ogawa, T.; Lkhamsuren, G.; Cho, N.-J.; Hayashi, T. Quantitative Evaluation of Peptide-Material Interactions by a Force Mapping Method: Guidelines for Surface Modification. Langmuir 2015, 31, 8006-8012.

(24) Ducker, W. A.; Senden, T. J.; Pashley, R. M. Measurement of Forces in Liquids Using a Force Microscope. Langmuir 1992, 8, 18311836.

(25) Ralston, J.; Larson, I.; Rutland, M. W.; Feiler, A. A.; Kleijn, M. Atomic Force Microscopy and Direct Surface Force Measurements. Pure Appl. Chem. 2005, 77, 2149-2170.

(26) Hayashi, T.; Tanaka, Y.; Koide, Y.; Tanaka, M.; Hara, M. Mechanism Underlying Bioinertness of Self-Assembled Monolayers of Oligo(ethyleneglycol)-Terminated Alkanethiols on Gold: Protein Adsorption, Platelet Adhesion, and Surface Forces. Phys. Chem. Chem. Phys. 2012, 14, 10196-10206.

(27) Demers, L. M.; Mirkin, C. A.; Mucic, R. C.; Reynolds, R. A., III; Letsinger, R. L.; Elghanian, R.; Viswanadham, G. A Fluorescent-Based Method for Determining the Surface Coverage and Hybridization Efficiency of Thiol-Capped Oligonucleotides Bound to Gold Thin Films and Nanoparticles. Anal. Chem. 2000, 72, 5535-5541.

(28) Monserud, J. H.; Schwartz, D. K. Mechanisms of SurfaceMediated DNA Hybridization. ACS Nano 2014, 8, 4488-4499.

(29) Zucker, M. Mfold Web Server for Nucleic Acid Folding and Hybridization Prediction. Nucleic Acids Res. 2003, 31, 3406-3415.

(30) Hurst, S. J.; Lytton-Jean, A. K. R.; Mirkin, C. A. Maximizing DNA Loading on a Range of Gold Nanoparticle Sizes. Anal. Chem. 2006, 78, 8313-8318.

(31) Buchner, R.; Hefter, G. T.; May, P. M. Dielectric Relaxation of Aqueous $\mathrm{NaCl}$ Solutions. J. Phys. Chem. A 1999, 103, 1-9.

(32) Tadmor, R.; Hernández-Zapata, E.; Chen, N.; Pincus, P.; Israelachvili, J. N. Debye Length and Double-Layer Forces in Polyelectrolyte Solutions. Macromolecules 2002, 35, 2380-2388.

(33) Zimmer, C.; Luck, G. Conformation and Reactivity of DNA III. Circular Dichroism Studies of the Effects of Aqueous Concentrated Univalent Salt Solutions upon Helix Conformation. Biochim. Biophys. Acta, Nucleic Acids Protein Synth. 1973, 312, 215-227.

(34) Wolf, B.; Hanlon, S. Structural Transitions of Deoxyribonucleic Acid in Aqueous Electrolyte Solutions. II. Role of Hydration. Biochemistry 1975, 14, 1661-1670.

(35) Maffeo, C.; Luan, B.; Aksimentiev, A. End-to-End Attraction of Duplex DNA. Nucleic Acids Res. 2012, 40, 3812-3821.

(36) Yan, H.; LaBean, T. H.; Feng, L.; Reif, J. H. Directed Nucleation Assembly of DNA Tile Complexes for Barcode-Patterned Lattices. Proc. Natl. Acad. Sci. U.S.A. 2003, 100, 8103-8108.

(37) Wang, R.; Kuzuya, A.; Liu, W.; Seeman, N. C. Blunt-Ended DNA Stacking Interactions in a 3-Helix Motif. Chem. Commun. 2010, 46, 4905-4907.

(38) Nakata, M.; Zanchetta, G.; Chapman, B. D.; Jones, C. D.; Cross, J. O.; Pindak, R.; Bellini, T.; Clark, N. A. End-to-End Stacking and Liquid Crystal Condensation of 6-to-20-Base Pair DNA Duplexes. Science 2007, 318, 1276-1279.

(39) Woo, S.; Rothemund, P. W. K. Programmable Molecular Recognition Based on the Geometry of DNA Nanostructures. Nat. Chem. 2011, 3, 620-627.

(40) Rafat, A. A.; Pirzer, T.; Scheible, M. B.; Kostina, A.; Simmel, F. C. Surface-Assisted Large-Scale Ordering of DNA Origami Tiles. Angew. Chem., Int. Ed. 2014, 53, 7665-7668.

(41) Suzuki, Y.; Endo, M.; Sugiyama, H. Lipid-Bilayer-Assisted TwoDimensional Self-Assembly of DNA Origami Nanostructures. Nat. Commun. 2015, 6, 8052.

(42) Kilchherr, F.; Wachauf, C.; Pelz, B.; Rief, M.; Zacharias, M.; Dietz, H. Single-Molecule Dissection of Stacking Forces in DNA. Science 2016, 353, aaf5508.

(43) Qiu, X.; Anderson, K.; Kwok, L. W.; Lamb, J. S.; Park, H. Y.; Pollack, L. Inter-DNA Attraction Mediated by Divalent Counterions. Phys. Rev. Lett. 2007, 99, 038104.

(44) Li, L.; Pabit, S. A.; Lamb, J. S.; Park, H. Y.; Pollack, L. Closing the Lid on DNA End-to-End Stacking Interactions. Appl. Phys. Lett. 2008, 92, 223901.
(45) Kanayama, N.; Takarada, T.; Maeda, M. Rapid Naked-Eye Detection of Mercury Ions Based on Non-Crosslinking Aggregation of Double-Stranded DNA-Carrying Gold Nanoparticles. Chem. Commun. 2011, 47, 2077-2079.

(46) Kanayama, N.; Takarada, T.; Fujita, M.; Maeda, M. DNA Terminal Breathing Regulated by Metal Ions for Colloidal Logic Gates. Chem.-Eur. J. 2013, 19, 10794-10798.

(47) Akiyama, Y.; Shikagawa, H.; Kanayama, N.; Takarada, T.; Maeda, M. Modulation of Interparticle Distance in Discrete Gold Nanoparticle Dimers and Trimers by DNA Single-Base Pairing. Small 2015, 11, 3153-3161.

(48) Akiyama, Y.; Shikagawa, H.; Kanayama, N.; Takarada, T.; Maeda, M. DNA Dangling-End-Induced Colloidal Stabilization of Gold Nanoparticles for Colorimetric Single-Nucleotide Polymorphism Genotyping. Chem.-Eur. J. 2014, 20, 17420-17425.

(49) Moore, R. D.; Morrill, G. A. A Possible Mechanism for Concentrating Sodium and Potassium in the Cell Nucleus. Biophys. J. $1976,16,527-533$. 